Revista Brasileira de Meteorologia, v.25, n.1, 24 - 31, 2010

\title{
DETERMINAÇÃO DO ALBEDO DA SUPERFÍCIE A PARTIR DE DADOS AVHRR/NOAA E TM/ LANDSAT-5
}

\author{
FABIANE REGINA DA CUNHA DANTAS, CÉLIA CAMPOS BRAGA, ENIO PEREIRA DE SOUZA, \\ SAULO TASSO ARAÚJO DA SILVA
}

\author{
Universidade Federal de Campina Grande - UFCG, Campina Grande - PB, Brasil \\ fabiane@dca.ufcg.edu.br, celia@dca.ufcg.edu.br, esouza@dca.ufcg.edu.br, saulo@funceme.br
}

Recebido Agosto 2008 - Aceito Agosto 2009

\begin{abstract}
RESUMO
Este trabalho teve como objetivo estimar uma distribuição espacial do albedo da superfície para obter uma relação funcional entre o albedo dos sensores AVHRR/NOAA e TM/LANDSAT-5, sobre diferentes alvos nas proximidades de Quixeré-CE, nos anos de 2005 e 2006. As imagens foram processadas pelo software Erdas Image 8.7 utilizando o SEBAL (Surface Energy Balance Algorithm for Land). Os resultados mostraram diferenças na variabilidade espacial e temporal do albedo nos dois anos estudados, pois 2005 foi predominantemente seco enquanto que 2006 foi um ano chuvoso. A relação funcional entre os dados AVHRR e TM apresentou coeficiente de correlação (r) igual a 0,75 com erro padrão de $1,67 \%$. Valores de albedo da superfície estimados pelo AVHRR e TM apresentaram respectivamente erros padrões de 2,44 e 3,45\%, quando comparados com as observações do radiômetro CNR1.
\end{abstract}

Palavras-Chave: sensoriamento remoto, SEBAL, regressão linear, Frutacor

\begin{abstract}
DETERMINATION OF SURFACE ALBEDO FROM AVHRR/NOAA AND TM/ LANDSAT-5 DATA

This work aims to estimate a spatial distribution of surface albedo and to derive a functional relationship between the albedo from AVHRR/NOAA and TM/LANDSAT-5 sensors over different targets in the proximities of Quixeré - CE during the years of 2005 and 2006. The images were processed by the software Erdas Image 8.7 using the SEBAL (Surface Energy Balance Algorithm for Land). The results showed differences in the spatial and temporal variability of the albedo in the two studied years, because 2005 was predominantly dry while 2006 was a rainy year. The functional relationship between AVHRR and TM data presented correlation coefficient (r) value equal to 0.75 with a standard error of $1.67 \%$. Surface albedo values estimated by AVHRR and TM showed standard errors of 2.44 and $3.45 \%$ respectively when compared to the CNR1 radiometer observations.
\end{abstract}

Keywords: remote sensing, SEBAL, linear regression, Frutacor

\section{INTRODUÇÃO}

Nas últimas décadas, os dados de sensoriamento remoto têm sido de fundamental importância em pesquisas direcionadas em estudar o comportamento dinâmico da vegetação, monitoramento de diversos fenômenos meteorológicos e ambientais, obtidos a partir de sensores a bordo de satélites, oferecendo grande suporte às previsões de tempo e clima. Eles constituem uma ferramenta de grande valia na obtenção de informações necessárias ao manejo, gerenciamento e gestão de recursos naturais, tais como água, solo e vegetação (Batista \& Almeida, 1998), como também na agricultura por oferecer diversas vantagens possibilitando a geração de séries temporais das cenas estudadas.

Dados multitemporais obtidos do sensoriamento remoto de diferentes satélites meteorológicos e ambientais 
têm sido amplamente utilizados com diferentes finalidades. Gutman \& Ignatov (1998) parametrizaram a fração da vegetação utilizando dados de IVDN (Índice de Vegetação por Diferença Normalizada), para serem incorporados em modelos de previsão numérica de tempo e clima. Os resultados preliminares mostraram uma melhora significativa na previsão dos fluxos à superfície. Melo (2003) utilizou dados de IVDN obtidos das imagens de satélites do AVHRR/NOAA para estimar o IAF (Índice de Área Foliar) e a fração da vegetação, na região Nordeste do Brasil, incorporando-os no modelo regional de previsão de tempo RAMS. A autora verificou que a substituição desses parâmetros no modelo resultou em mudanças significantes nos fluxos de calor sensível e latente. Silva et al. (2005) determinaram o albedo da superfície em áreas irrigadas na região do Vale do São Francisco utilizando imagens do satélite LANDSAT- 5 encontrando valores do albedo entre 17 e $20 \%$ para pomares de frutíferas irrigadas. Braga et al. (2006) utilizaram dados do AVHRR/NOAA para calcular a fração vegetação anual nas décadas de 1980 e 1990 no leste da Bahia, constatando que na década de 1980 ocorreu redução da vegetação Caatinga e aumento da vegetação no Agreste e Mata Atlântica, enquanto que na década de 1990 a vegetação apresentou redução na região do Agreste e Mata Atlântica e aumento na Caatinga. Houborg et al. (2007) desenvolveram um método de otimização numérica utilizando um modelo de inversão da refletância no dossel da vegetação, combinando índices de vegetação e parâmetros biofísicos obtido pelo sensor MODIS Aqua-Terra na Dinamarca compreendendo áreas de cultivo agrícola e florestas coníferas e de folhas largas. Os resultados mostraram, que a relação entre o IAF medido e o estimado em cultivo de cevada, trigo e floresta, explicaram 62, 46 e $63 \%$ da variância total dos dados, respectivamente.

Considerando a gama de trabalhos envolvendo dados obtidos remotamente e a carência de estudos com esse enfoque, o objetivo deste estudo é avaliar a variabilidade espacial do albedo da superfície sobre diferentes alvos na região de Quixeré-CE,

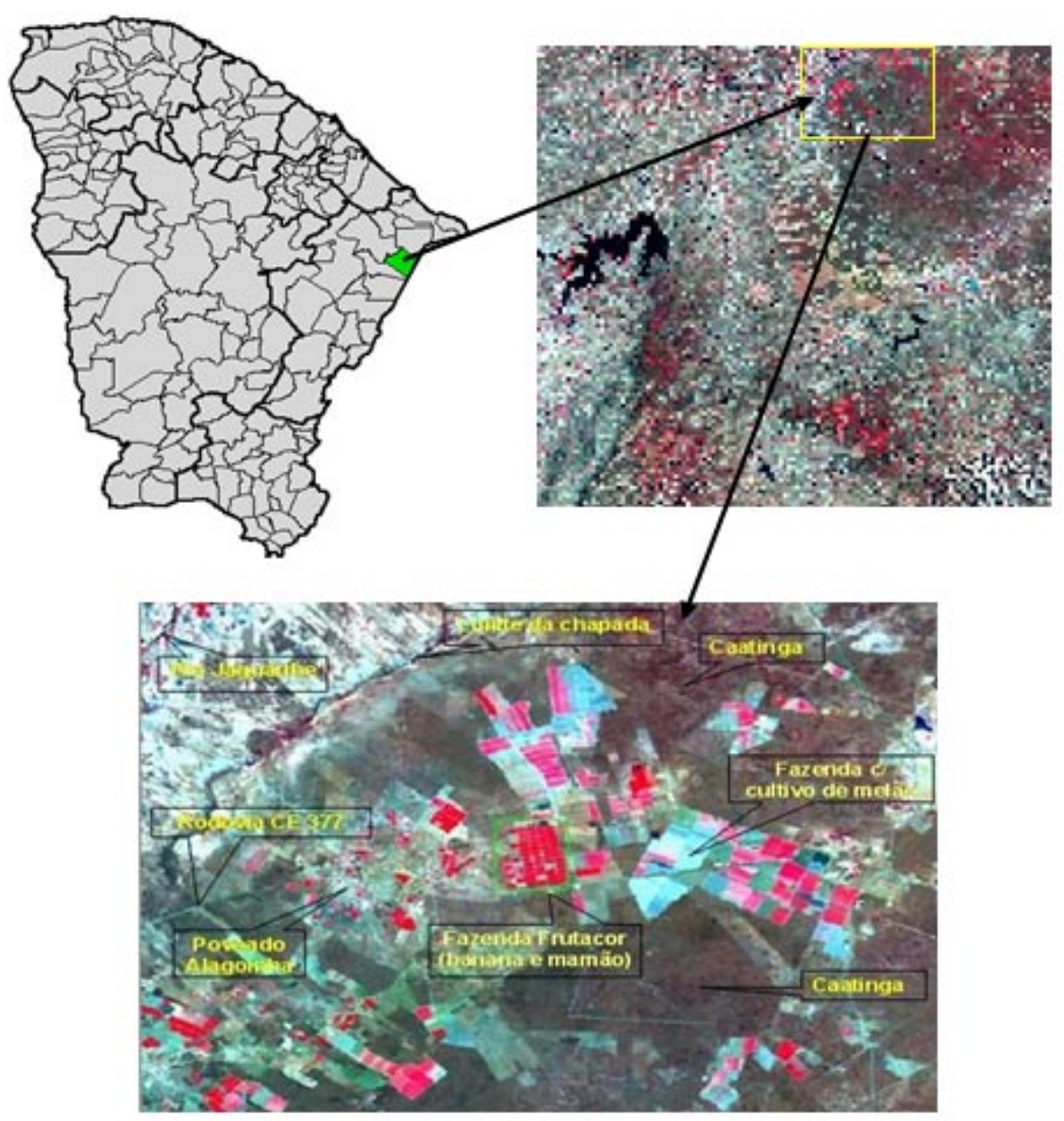

Figura 1. Composição das bandas do Mapeador temático do satélite Landsat 5 e localização da área de estudo próxima a Quixeré - CE 
e encontrar uma relação funcional entre o albedo obtido pelos sensores AVHRR e TM e medidos sobre o pomar de bananeiras nos anos de 2005 e 2006.

\section{MATERIAL E MÉTODOS}

Foram utilizadas nesta pesquisa cinco imagens do sensor TM (Thematic Mapper) do satélite LANDSAT-5, com órbita/ ponto 216/064 convertidas para $1 \mathrm{~km}$ de resolução, composta de sete canais adquirida ao INPE e cinco do AVHRR (Advanced Very High Resolution Radiometer) do NOAA, composta de 5 canais, entre as latitudes $\left(5^{\circ} 02\right.$ ' $51^{\prime \prime} \mathrm{S}$ e $5^{\circ} 02^{\prime} 56^{\prime \prime} \mathrm{S} ; 6^{\circ} 17^{\prime} 10^{\prime \prime}$ $\mathrm{S}$ e $6^{\circ} 17^{\prime} 05^{\prime}$ " S) e longitudes ( $38^{\circ} 41^{\prime} 59^{\prime \prime} \mathrm{W}$ e $37^{\circ} 28^{\prime} 26^{\prime \prime} \mathrm{W}$; $38^{\circ} 37^{\prime} 51^{\prime \prime}$ W e $37^{\circ} 28^{\prime} 46^{\prime \prime} \mathrm{W}$ ) para os dias 21 de agosto de 2005,24 de outubro de 2005, 23 de julho de 2006, 08 de agosto de 2006 e 24 de agosto de 2006.

A região de estudo compreende uma área de $15.225 \mathrm{~km}^{2}$ situada no leste do Ceará nas proximidades de Quixeré, áreas de agricultura irrigada (Fazenda Frutacor) e áreas de vegetação nativa conforme indicado na Figura 1.

Os dados medidos do albedo da superfície foram obtidos de um experimento de campo iniciado em agosto de $2005 \mathrm{com}$ término em setembro de 2006, realizado próximo a cidade de Quixeré - CE, utilizando o radiômetro CNR1 (Campbell Scientific, Inc, Reino Unido) para computar as medidas individuais dos sensores de ondas curtas e ondas longas. $\mathrm{O}$ CNR1 opera em toda faixa do visível ( 0,3 a 3 micrometros) e do infravermelho (5 a 50 micrometros), com erro instrumental de aproximadamente de $2,5 \%$.

O processamento das imagens AVHRR/NOAA e TM/ LANDSAT foi desenvolvido através da ferramenta Model Maker do software ERDAS Image 8.7 utilizando o algoritmo SEBAL, que é um método semi-empírico, o qual promove a parametrização do balanço de energia e fluxos de superfície baseado em alguns dados locais e medições espectrais de satélites (Weligepolage, 2005). As etapas utilizadas para o cálculo do albedo são descritas a seguir:

i) Calibração radiométrica é o processo de conversão do número digital (ND) em radiância espectral monocromática $L_{\lambda i}$. Para as bandas refletivas do LANDSAT-5 (canais 1, 2, 3, 4, 5 e 7) a calibração foi feita pela equação proposta por Markham \& Baker (1987):

$$
L_{\lambda i}=a_{i}+\frac{b_{i}-a_{i}}{255} N D
$$

em que, $a_{i}$ e $b_{i}$ são respectivamente as radiâncias espectrais mínimas e máximas $\left(\mathrm{Wm}^{-2} \mathrm{sr}^{-1} \mu \mathrm{m}^{-1}\right), N D$ é a intensidade do pixel (número inteiro de 0 a 255), $i=1, \ldots, 7$, corresponde às bandas do TM / LANDSAT. ii) Refletância espectral é a proporção entre o fluxo de radiação incidente numa superfície e o fluxo que é refletido. Foi utilizada para obtenção da refletância das imagens LANDSAT a equação de Allen et al. (2002) dada por:

$$
\rho_{T M, \lambda i}=\frac{\pi \times L_{\lambda i}}{k_{\lambda i} \times \cos Z \times d_{r}}
$$

em que $L_{\lambda i}$ é a radiância espectral da cada banda, $\mathrm{k}_{\lambda \mathrm{i}}$ é a irradiância solar espectral de cada banda no topo da atmosfera $\left(\mathrm{Wm}^{-2} \mathrm{sr}^{-1} \mu \mathrm{m}^{-1}\right)$, Z é o ângulo zenital solar e $d_{r}$ é o quadrado da razão entre a distância média Terra-Sol $\left(\mathrm{r}_{0}\right)$ e a distância TerraSol (r) em dado dia do ano. Para o AVHRR as refletâncias foram obtidas a partir dos coeficientes de calibração (inclinação $\mathrm{S}$ e intercepto I) contidos em arquivos brutos utilizando a expressão proposta em NOAA (2007):

$$
\rho_{A V H R R, \lambda i}=r^{2}\left(S_{i} \times C_{10, i}+I_{i}\right)
$$

em que $\rho_{A V H R R, \lambda i}$ é a refletância (\%) do pixel correspondente à banda $\mathrm{i}$ ( 1 ou 2 do AVHRR), $r$ é a distância Terra - Sol no dia em questão, $C_{10, i}$ é a intensidade no pixel (em 10 bits) e $S_{i}$ e $I_{i}$ são os coeficientes de calibração da banda i.

iii) Albedo Planetário, isto é, refletâncias espectrais medidas no topo da atmosfera não ajustado à transmissividade atmosférica foi obtido pelas expressões propostas por Hucek \& Jacobowitz (1995) em que:

$$
\alpha_{\text {AVHRR }}=0,40 \times \rho_{1}+0,43 \times \rho_{2}+2,2
$$

onde $\rho_{1}(\%)$ e $\rho_{2}$ (\%) são as refletâncias das bandas 1 e 2 do AVHRR e Bastiaanssen (1995) onde:

$$
\begin{aligned}
\alpha_{\mathrm{TM}}= & 0,293 \times \rho_{1}+0,274 \times \rho_{2}+0,233 \times \rho_{3} . \\
& +0,157 \times \rho_{4}+0,033 \times \rho_{5}+0,011 \times \rho_{7}
\end{aligned}
$$

em que $\rho_{1}, \rho_{2}, \rho_{3}, \rho_{4}, \rho_{5}$ e $\rho_{7}$ são os albedos planetários das bandas $1,2,3,4,5$ e 7 do TM/LANDSAT.

iv) O albedo da superfície foi dado pela equação de Bastiaanssen (1995):

$$
\alpha_{c o}=\frac{(\alpha-a)}{b}
$$

em que $\alpha$ é o albedo planetário e os coeficientes $a$ e $b$ representam respectivamente, a refletância atmosférica para a radiação de onda curta e o quadrado da transmitância atmosférica de onda curta que pode ser obtida por:

$$
\tau_{S W}=0,75+2 \cdot 10^{-5} \mathrm{z}
$$

em que $\mathrm{z}$ representa a altitude de cada pixel. 
$\mathrm{Na}$ análise estatística dos dados para encontrar a relação existente entre o albedo estimado pelo AVHRR/NOAA e pelo TM/LANDSAT, utilizou-se o método da regressão linear segundo critério dos Mínimos Quadrados. Maiores detalhes da metodologia encontram-se em Spiegel (1968), Wilks (1995).

\section{RESULTADOS}

\subsection{Albedo a Superfície}

As Figuras $2 \mathrm{a}$ - $2 \mathrm{j}$ representam a distribuição espacial e temporal do albedo da superfície para 2005 e 2006. Nas áreas estudadas fez-se uma composição de cores das bandas dos dois satélites para os diferentes alvos imageados pelos sensores, a fim de faciltar o entendimento e análise dos resultados. Os maiores valores de albedo ocorreram nos meses de agosto e outubro de 2005 por causa da estiagem neste período. A cor azul escura nas figuras indica menores valores do albedo, variando entre 4 e $13 \%$, correspondendo a reservatórios de água e solo úmido. Valores superiores a $34 \%$ indicam solo pouco vegetado e topo de nuvens (cor azul clara). No nordeste da região, fazenda Frutacor, área frutífera irrigada o albedo variou entre 13 e $20 \%$ em quase todos os meses, devido à irrigação, à vegetação mais verde $\mathrm{e}$ ao menor albedo. Em áreas de vegetação nativa localizadas na

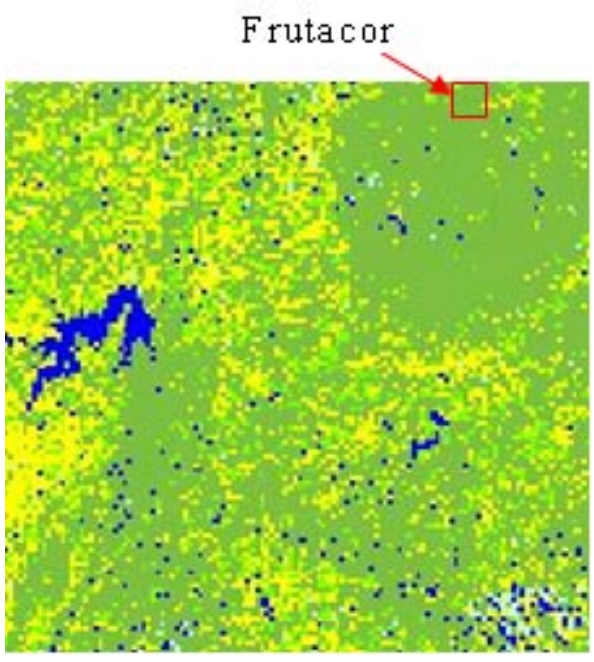

(a) 21-08-05 - LANDSAT

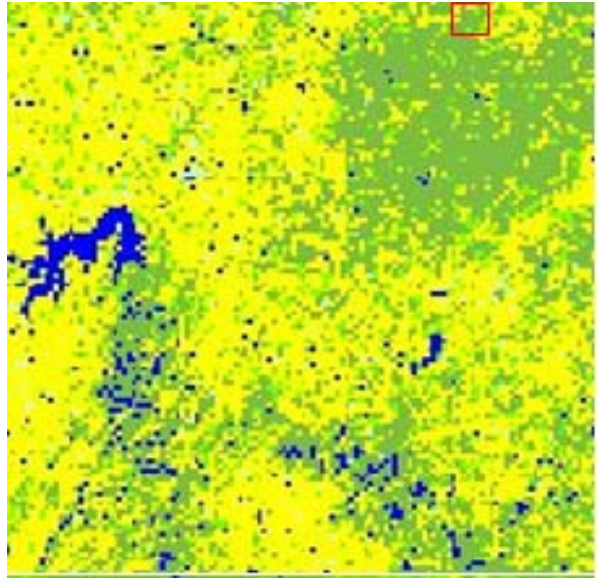

(c) 24-10-05 - LANDSAT

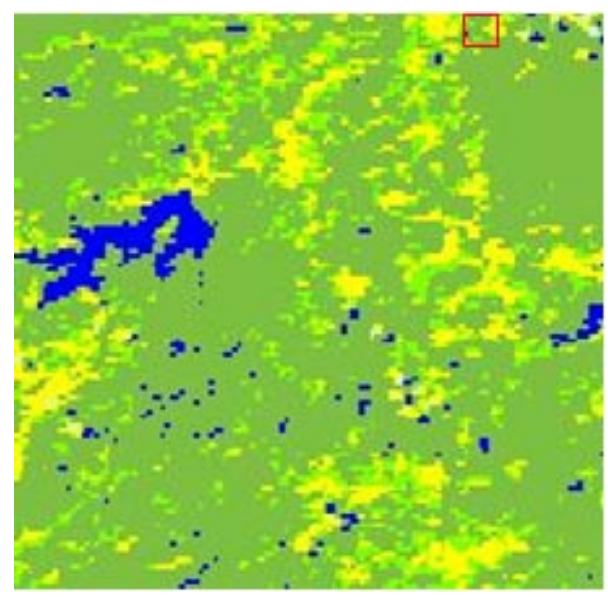

(b) 21-08-05 - NOAA

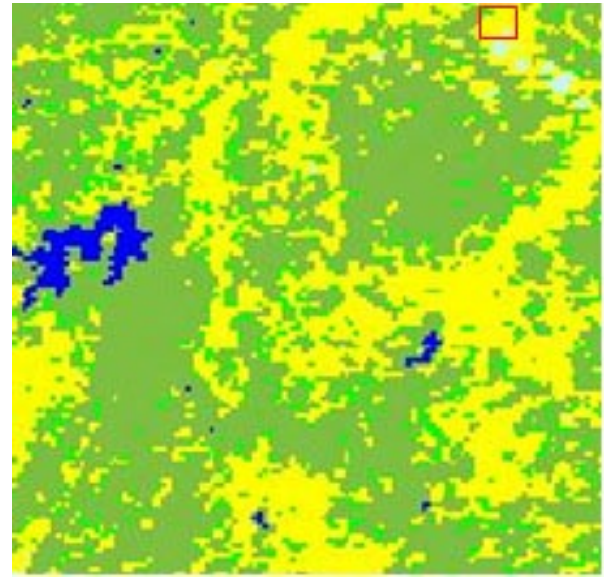

(d) 24-10-05 - NOAA

Figura 2 (a, b, $, \mathbf{c}, \mathbf{d}, \mathbf{e}, \mathbf{f}, \mathbf{g}, \mathbf{h}, \mathbf{i}, \mathbf{j})$ - Distribuição espacial do Albedo a Superfície (LANDSAT / NOAA) na região de Quixeré e proximidades para 2005 e 2006 


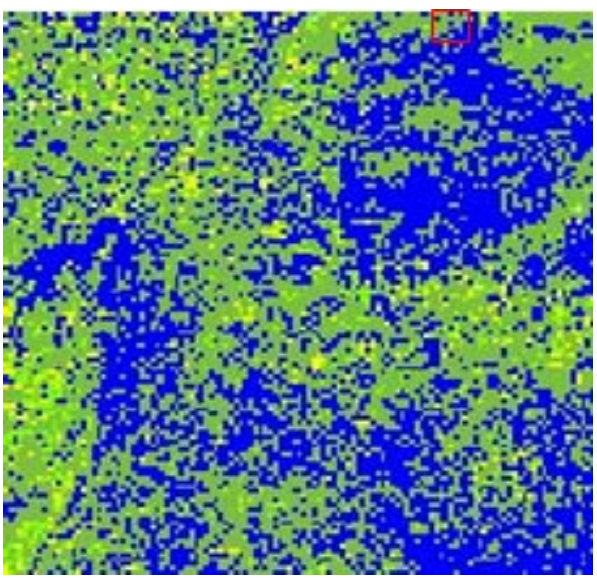

(e) 23-07-06 - LANDSAT

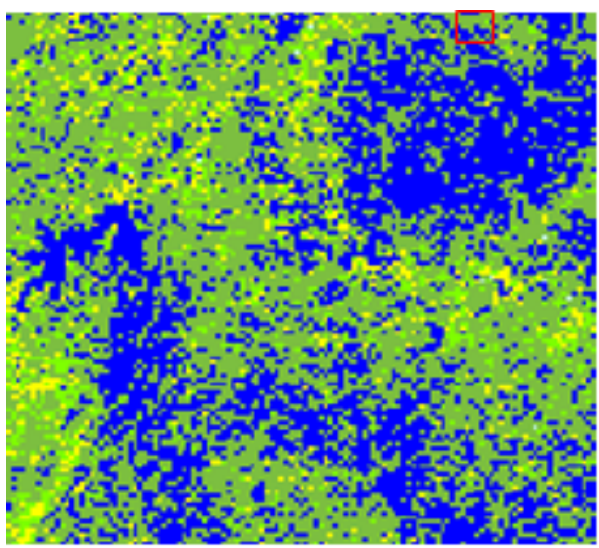

(g) 08-08-06 - LANDSAT

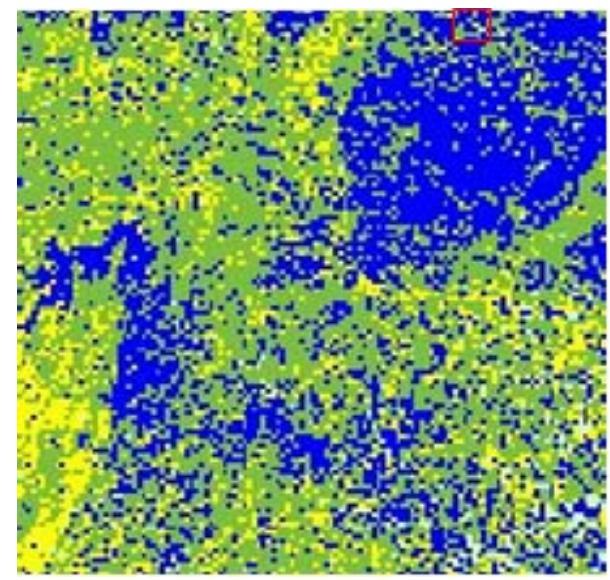

(i) 24-08-06 - LANDSAT

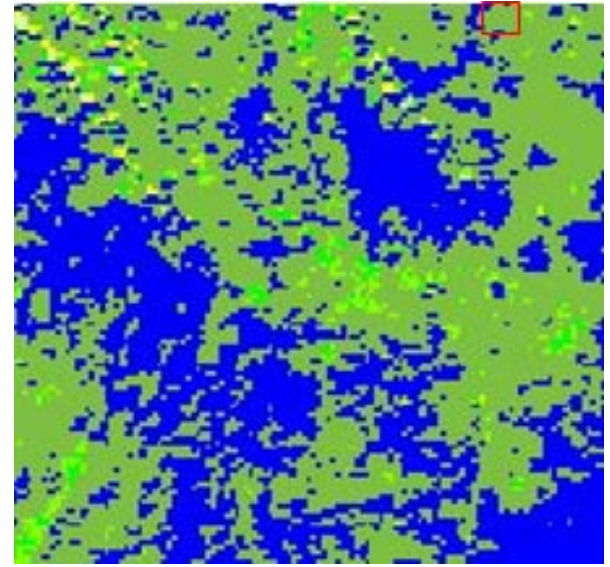

(f) 23-07-06 - NOAA

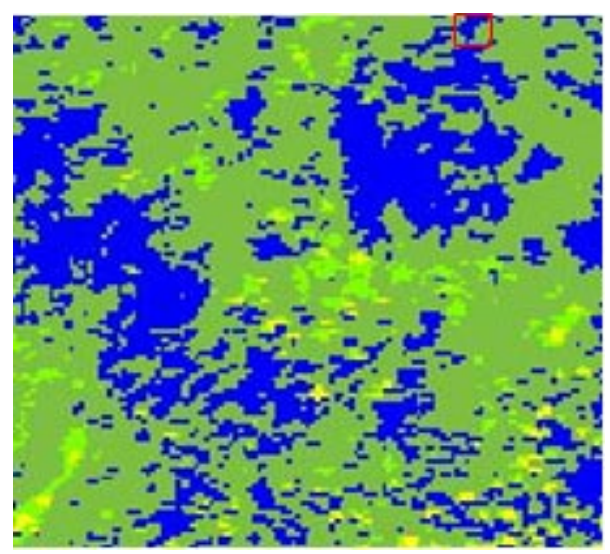

(h) 08-08-06 - NOAA

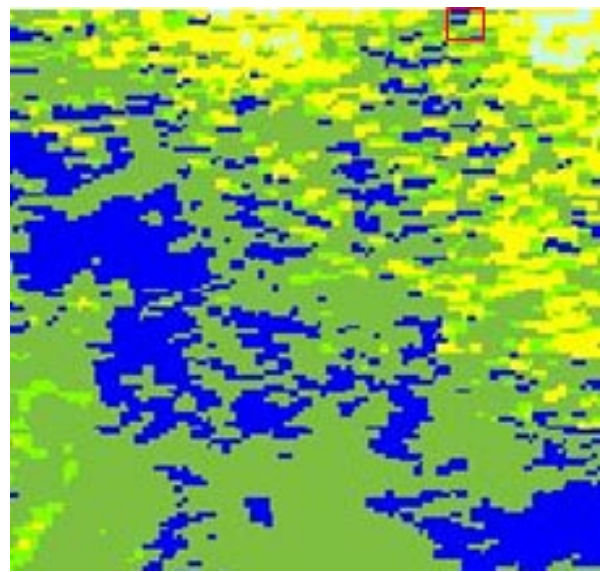

(j) 24-08-06 - NOAA 
diagonal noroeste-sudeste, e parte do nordeste e sudoeste da região, a variabilidade foi pequena oscilando entre 20 e $22 \%$. Para a caatinga (cor amarela) que ocupa grande parte da região de estudo, o albedo variou entre 20 e $34 \%$.

Para julho e agosto de 2006, observaram-se albedos menores variando de 4 a $22 \%$ em quase toda área. Observouse uma diferença considerável do albedo entre o ano de 2005 e 2006 nas vizinhanças da fazenda. Isto pode estar associado a maior quantidade de precipitação em junho, com média mensal de 130,2 mm em 2006 contra 71,6 mm em 2005 (FUNCEME, 2008), favorecendo o aumento da umidade do solo, e conseqüentemente, diminuindo o valor do albedo. Os resultados obtidos do albedo neste estudo concordam com os encontrados por Silva et al. (2005) em áreas irrigadas de frutíferas e Caatinga na região do vale do Rio São Francisco (Bahia/Pernambuco), os quais variaram entre 15 a 23\% e 20 a $30 \%$, respectivamente. Iziomons \& Mayer (2002) avaliando o comportamento do albedo em áreas de pastagens no sudoeste da Alemanha também obtiveram albedo variando entre 22,2 a $36 \%$.

Na Figura 3 e Tabela 1 são mostrados a dispersão e a reta de regressão dos mínimos quadrados, para os valores médios do albedo obtido das cinco imagens LANDSAT e NOAA para os anos de 2005 e 2006, na fazenda Frutacor e vizinhança do município de Quixeré-CE para os diferentes alvos: Frutíferas irrigadas, Caatinga e área mista (Caatinga e agricultura irrigada).

A equação de regressão obtida para os alvos é igual a: y $=0,53 \mathrm{x}+0,06 . \mathrm{O}$ erro padrão da estimativa foi de $1,67 \% \mathrm{com}$ coeficiente de correlação linear igual a 0,75 , o que implica num coeficiente de determinação $\mathrm{r}^{2}=0,56$. O modelo linear explica, portanto, $56 \%$ da variância total dos dados. A significância do coeficiente de correlação linear (r) foi baseada no teste de Student (t), para o nível de significância de $\alpha=0,01$, com 13 graus de liberdade da amostra $(v)$, o que resultou em $t_{\text {tabelado }}=2,65$ contra $t_{\text {cal }}=4,08$. Os resultados indicam que a hipótese nula de não existência de correlação entre o albedo inferidos através de TM e AVHRR, pode ser rejeitada ao nível de significância de $1 \%$ em favor a hipótese alternativa.

Observando o coeficiente de correlação verificou-se que neste conjunto de dados médios de vegetação composta por Frutífera, Caatinga e área mista, 56\% das variações encontradas no albedo TM do modelo linear, são explicados pela variável independente (albedo AVHRR). Diante desses resultados observa-se que na falta do sensor de alta resolução pode-se utilizar os dados de sensor de menor resolução, como o AVHRR, com cautela dependo da finalidade.

Os resultados encontrados foram satisfatórios mesmo levando-se em consideração o fato da capacidade do sensor AVHRR (resolução espacial de $1 \mathrm{~km}$ ) ser pouco adequado à identificação de determinados alvos. É evidente que o sensor TM de resolução espacial mais fina $(30 \mathrm{~m})$ associado a uma ampla resolução espectral, permite melhor caracterização de áreas pequenas com diferentes alvos, facilitando assim a identificação mais precisa da cobertura.

\subsection{Comparação entre o Albedo Medido e o Calculado pelo SEBAL}

Na Tabela 2, estão apresentados os valores do albedo da superfície para quatro dias selecionados nos anos de 2005 e 2006. Estes valores referem-se ao albedo medido com radiômetro, seus

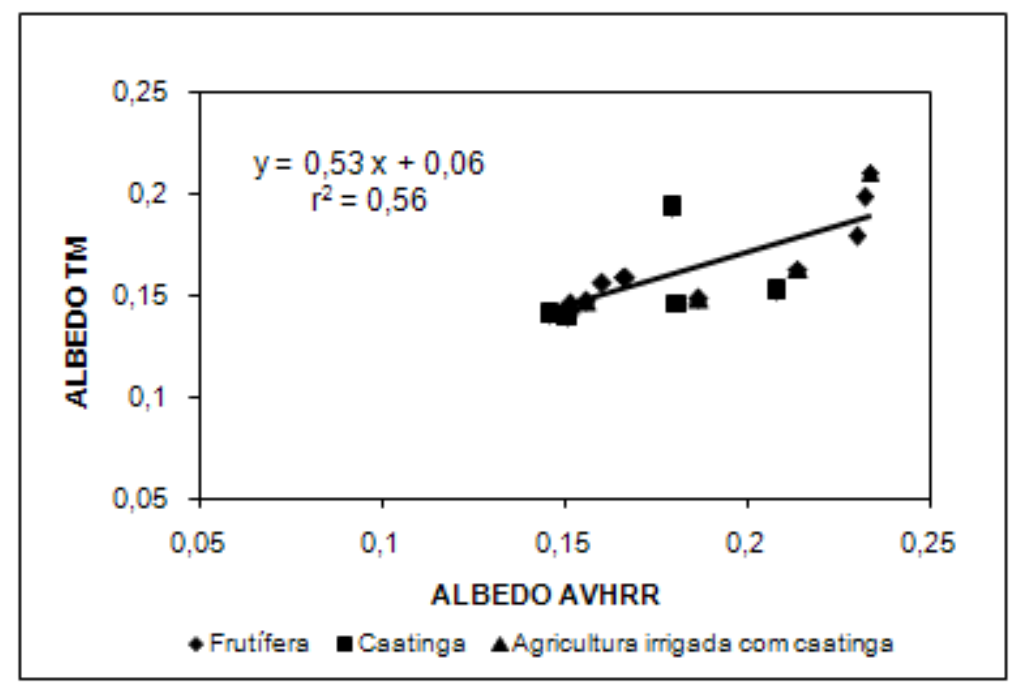

Figura 3 - Relação funcional do albedo médio obtido pelo AVHRR e TM para áreas de Frutífera, Caatinga e área mista (Caatinga e agricultura irrigada). 
respectivos desvios padrões e o calculado pelo SEBAL, num ponto localizado na área da fazenda Frutacor (Figura 1).

Para os valores medidos no pomar de bananeiras (latitude $5^{\circ} 04^{\prime} 35^{\prime}$ 'S e longitude $37^{\circ} 51^{\prime} 54^{\prime}$ ' W) (Figura 1), os do albedo medidos para as quatro datas analisadas, oscilaram entre 0,161 e 0,169 com valor médio instantâneo de 0,166 \pm 0,031 . Observa-se que as diferenças entre os valores medidos e calculados (AVHRR e TM) foram pequenas, com erros padrões de estimativa de aproximadamente 2,44 e $3,45 \%$, respectivamente. Os valores do albedo medidos são inferiores a $17 \%$ para todos os dias estudados, enquanto os estimados pelos sensores AVHRR e TM não ultrapassaram os 23 e $21 \%$, respectivamente. É evidente que essas diferenças podem estar relacionadas com os efeitos atmosféricos, diferença no horário de passagem dos satélites, assim como presença de nuvens. Observa-se que para imagem do dia $24 / 10$ os valores estimados estão afastados da margem de erro, possivelmente por contaminação de nuvem no ponto de medição (Figura 2d). Esses

Tabela 1. Valores médios de albedo para LANDSAT e NOAA obtidos para Frutífera, Caatinga e Mista.

\begin{tabular}{|c|c|c|c|}
\hline \multicolumn{4}{|c|}{ Albedo } \\
\hline Vegetação & Dia & LANDSAT & NOAA \\
\hline \multirow{4}{*}{ Frutífera } & $21 / 08 / 05$ & 0,18 & 0,23 \\
\cline { 2 - 4 } & $24 / 10 / 05$ & 0,20 & 0,23 \\
\cline { 2 - 4 } & $23 / 07 / 06$ & 0,16 & 0,16 \\
\cline { 2 - 4 } & $08 / 08 / 06$ & 0,16 & 0,17 \\
\cline { 2 - 4 } & $24 / 08 / 06$ & 0,16 & 0,17 \\
\hline \multirow{5}{*}{ Maatinga } & $21 / 08 / 05$ & 0,15 & 0,21 \\
\cline { 2 - 4 } & $24 / 10 / 05$ & 0,19 & 0,18 \\
\cline { 2 - 4 } & $23 / 07 / 06$ & 0,14 & 0,15 \\
\cline { 2 - 4 } & $08 / 08 / 06$ & 0,14 & 0,15 \\
\cline { 2 - 4 } & $24 / 08 / 06$ & 0,15 & 0,18 \\
\hline \multirow{5}{*}{ Mista } & $21 / 08 / 05$ & 0,16 & 0,21 \\
\cline { 2 - 4 } & $24 / 10 / 05$ & 0,21 & 0,23 \\
\cline { 2 - 4 } & $23 / 07 / 06$ & 0,15 & 0,15 \\
\cline { 2 - 4 } & $08 / 08 / 06$ & 0,15 & 0,16 \\
\cline { 2 - 4 } & $24 / 08 / 06$ & 0,15 & 0,19 \\
\hline & & & \\
\hline
\end{tabular}

Tabela 2. Comparação entre os valores medidos e estimados do albedo pelo SEBAL

\begin{tabular}{|c|c|c|c|c|}
\hline Erutacor & \multicolumn{4}{|c|}{ Albedo da Superfície } \\
\hline & Medido & $\mathbf{X} \pm \boldsymbol{\sigma}_{\mathbf{x}}$ & LANDSAT & NOAA \\
\hline $24 / 10 / 2005$ & 0,165 & $0,165 \pm 0,0031$ & 0,210 & 0,232 \\
\hline $23 / 07 / 2006$ & 0,161 & $0,161 \pm 0,0031$ & 0,163 & 0,156 \\
\hline $08 / 08 / 2006$ & 0,169 & $0,169 \pm 0,0031$ & 0,169 & 0,157 \\
\hline $24 / 08 / 2006$ & 0,168 & $0,168 \pm 0,0031$ & 0,149 & 0,158 \\
\hline
\end{tabular}


valores estão em concordância com os obtidos por Song et al. (1999) sobre vegetações de pastagem e agricultura em diferentes locais nos EUA, utilizando distintos métodos para estimar o albedo. Sendo que dos métodos utilizados o que apresentou menor erro de $2,2 \%$ foi o desenvolvido pelos autores, com o albedo variando entre 12 e $28 \%$. Silva et al. (2005) também obtiveram albedos variando entre 15 e $25 \%$ em áreas irrigadas na região do vale do São Francisco.

\section{CONCLUSÕES}

A análise espacial e temporal do albedo à superfície possibilitou encontrar relações funcionais entre os dados do AVHRR e TM, através do modelo de regressão de mínimos quadrados sobre os diferentes alvos estudados, mostrando um relacionamento significativo $(\mathrm{r}=0,75)$ entre as informações, com erro padrão de estimativa da ordem de $1,67 \%$.

$\mathrm{O}$ albedo variou bastante de um ano a outro, mesmo nas áreas irrigadas. Os maiores valores registrados verificaram-se nos meses de agosto e outubro de 2005.

Os valores de albedo medidos e estimados pelos dois satélites na fazenda Frutacor foram bem coerentes apresentando erros inferiores a $4 \%$.

Diante dos resultados obtidos é possível a utilização com cautela dos dados NOAA em lugar do LANDSAT, principalmente em modelos de previsão de tempo e clima, porém para uma definição mais precisa é aconselhável usar satélites de maior resolução espacial, como por exemplo, o LANDSAT.

\section{REFERÊNCIAS BIBLIOGRÁFICAS}

ALLEN, R. G; TREZZA, R.; TASUMI, M. Surface Energy

Balance Algorithms for Land. Advance training and users manual, version 1.0, pp.98, 2002.

BASTIAANSSEN, W. G. M. Regionalization of surface flux densities and moisture indicators in composite terrain. $\mathrm{Ph}$. D. Thessis, Wageningen Agricultural University, Wageningen, The Netherlands, 273f, 1995.

BATISTA, G. T; ALMEIDA, E. S. Índice de Vegetação versus precipitação na Amazônia. In: SIMPÓSIO BRASILEIRO DE SENSORIAMENTO REMOTO, 9., Santos, 1998. Anais... INPE, 1998. CD-ROM.

BRAGA, C. C.; NEVES, D. G.; BRITO, J. I. Estimativa da fração vegetação no leste da Bahia no período de 1982 a 1999. Revista Brasileira de Agrometeorologia, v. 14, n. 3, p. $252-260,2006$.

FUNDAÇÃO CEARENSE DE METEOROLOGIA E RECURSOS HÍDRICOS - FUNCEME. Disponível em: Www.funceme.br. Acesso em: 15 Jan. 2008.

GUTMAN, G.; IGNATOV, A. The derivation of the green vegetation fraction from NOAA/AVHRR data for use in numerical weather prediction models. International Journal. Remote Sensing. v.19, n.8, 1533-1543, 1998.

HOUBORG, R.; SOEGAARD, H.; BOEGH, E. Combining Vegetation Index and Model Inversion Methods for the Extraction of Key Vegetation Biophysical parameters using Terra and Aqua MODIS reflectance Data. Remote Sensing of Enviroment. 106, 39-58, 2007.

HUCEK, R.; JACOBOWITZ, H. Impact of scene dependenc e on AVHRR albedo models. Journauls of Atmospheric and Oceanic Tecnology. v.12, n.4. 697-711, 1995.

IZIOMONS, M. G \& MAYER, H. On the variability and modeling of surface albedo and long-wave radiation components. Agricultural and Forest Meteorology. v11. 141-152, 2002.

MARKHAM, B. L. \& BARKER, J. B. Thematic mapper band pass solar exoatmospherical irradiances. International Journal of Remote Sensing. v. 8, n. 3, p. 517-523, 1987.

MELO, M. L. D. Efeito da vegetação em simulações numéricas com o modelo RAMS. 2003. $115 \mathrm{f}$. Dissertação (Mestrado em Meteorologia) Universidade Federal de Campina Grande, Campina Grande, 2003.

NATIONAL OCEANIC AND ATMOSPHERIC ADMINISTRATION - NOAA. Disponível em: http:// www2.ncdc.noaa.gov/docs $/ \mathrm{k} / \mathrm{m} / \mathrm{html} / \mathrm{c} 7 / \mathrm{sec} 7-1 . \mathrm{htm}$. Acesso em 20 Nov. 2007.

SILVA, B., B.; LOPES, M. L.; AZEVEDO, P. V. Determinação do albedo de áreas irrigadas com base em imagens LANDSAT 5 - TM. Revista Brasileira de Agrometeorologia. v. 13, n.2, 201-211, 2005.

SONG, J. \& GAO, W. An improved method to derive surface albedo from narrowband AVHRR satellite data: narrowband to broadband conversion. Journal of Applied Meteorology. V.8, 239-249, 1999.

SPIEGEL, M. R. Resumo de Teoria e Problemas de Estatística. Rio de Janeiro: Coleção Schaum, Livro Técnico - SA, 1968.

WELIGEPOLAGE, K. Estimation of spatial and temporal distribution of evapotranspiration bay satellite remote sensing - A case study in Hupselse Beek, The Netherlands, p.114, 2005.

WILKS, D. S. Statistical methods in the atmospheric sciences: and introduction. London: Academy Press, 1995. 464p. 